\title{
RELIABILITY FOR SOME BIVARIATE BETA DISTRIBUTIONS
}

\author{
SARALEES NADARAJAH
}

Received 24 June 2004

In the area of stress-strength models there has been a large amount of work as regards estimation of the reliability $R=\operatorname{Pr}(X<Y)$. The algebraic form for $R=\operatorname{Pr}(X<Y)$ has been worked out for the vast majority of the well-known distributions when $X$ and $Y$ are independent random variables belonging to the same univariate family. In this paper, we consider forms of $R$ when $(X, Y)$ follows a bivariate distribution with dependence between $X$ and $Y$. In particular, we derive explicit expressions for $R$ when the joint distribution is bivariate beta. The calculations involve the use of special functions.

\section{Introduction}

Bivariate beta distributions are tractable "lifetime" models in many areas, including life testing and telecommunications. In the context of reliability, the stress-strength model describes the life of a component which has a random strength $Y$ and is subjected to random stress $X$. The component fails at the instant that the stress applied to it exceeds the strength and the component will function satisfactorily whenever $Y>X$. Thus, $R=$ $\operatorname{Pr}(X<Y)$ is a measure of component reliability. It has many applications especially in engineering concepts, such as structures, deterioration of rocket motors, static fatigue of ceramic components, fatigue failure of aircraft structures, and the aging of concrete pressure vessels. Two examples are

(i) the receptor of a communication system operates only if it is stimulated by a source where magnitude $Y$ is greater than a random lower threshold $X$ for the system. In this case, $R$ is the probability that the receptor operates,

(ii) if $X$ and $Y$ are future observations on the stability of an engineering design, then $R$ would be predictive probability that $X$ is less than $Y$. Similarly, if $X$ and $Y$ represent lifetimes of two electronic devices, then $R$ is the probability that one fails before the other.

Because of these applications, the calculation and the estimation of $R=\operatorname{Pr}(X<Y)$ is important for the class of bivariate beta distributions. The calculation of $R$ has been extensively investigated in the literature when $X$ and $Y$ are independent random variables belonging to the same univariate family of distributions. The algebraic form for $R$ has been 
worked out for the majority of the well-known distributions, including normal, uniform, exponential, gamma, beta, extreme value, Weibull, Laplace, logistic, and the Pareto distributions (Nadarajah [5, 6, 7, 8, 9]; Nadarajah and Kotz [10]). In this paper, we calculate $R$ when $X$ and $Y$ are dependent random variables from five flexible families of bivariate beta distributions.

We will assume throughout this paper that $(X, Y)$ has a bivariate beta distribution with joint probability density function (pdf) $f$ and joint survivor function $\bar{F}$. One can write

$$
R=\int_{0}^{\infty} \int_{x}^{\infty} f(x, y) d y d x
$$

Our calculations of $R$ make use of a number of special functions. They are the incomplete beta function ratio defined by

$$
I_{x}(a, b)=\frac{1}{B(a, b)} \int_{0}^{x} t^{a-1}(1-t)^{b-1} d t ;
$$

the Gauss hypergeometric function $\left({ }_{2} F_{1}\right)$ defined by

$$
{ }_{2} F_{1}(a, b ; c ; x)=\sum_{k=0}^{\infty} \frac{(a)_{k}(b)_{k}}{(c)_{k}} \frac{x^{k}}{k !} ;
$$

and, the generalized hypergeometric function $\left({ }_{3} F_{2}\right)$ defined by

$$
{ }_{2} F_{2}(a, b, c ; d, e ; x)=\sum_{k=0}^{\infty} \frac{(a)_{k}(b)_{k}(c)_{k}}{(d)_{k}(e)_{k}} \frac{x^{k}}{k !},
$$

where $(f)_{k}=f(f+1) \cdots(f+k-1)$ denotes the ascending factorial. The properties of these special functions being used can be found in $[2,11,12,13]$.

\section{Dirichlet distribution}

Dirichlet distribution has the joint pdf specified by

$$
f(x, y)=K x^{a-1} y^{b-1}(1-x-y)^{c-1}
$$

for $x \geq 0, y \geq 0, x+y \leq 1, a>0, b>0$, and $c>0$, where

$$
K=\frac{\Gamma(a+b+c)}{\Gamma(a) \Gamma(b) \Gamma(c)} .
$$

The reliability in (1.1) can be expressed as

$$
\begin{aligned}
R & =K \int_{0}^{1 / 2} x^{a-1} \int_{x}^{1-x} y^{b-1}(1-x-y)^{c-1} d y d x \\
& =K \int_{0}^{1 / 2} x^{a-1}(1-x)^{b+c-1} \int_{x /(1-x)}^{1} z^{b-1}(1-z)^{c-1} d z d x \\
& =K B(c, b) \int_{0}^{1 / 2} x^{a-1}(1-x)^{b+c-1} I_{(1-2 x) /(1-x)}(c, b) d x
\end{aligned}
$$


where the transformation $z=y /(1-x)$ has been applied. Using the relationship

$$
I_{z}(\alpha, \beta)=\frac{z^{\alpha}}{\alpha B(\alpha, \beta)}{ }_{2} F_{1}(\alpha, 1-\beta ; \alpha+1 ; z),
$$

(2.3) can be rewritten as

$$
\begin{aligned}
R & =\frac{K}{c^{2}} \int_{0}^{1} x^{a-1}(1-x)^{b-1}(1-2 x)^{c}{ }_{2} F_{1}\left(c, 1-b ; c+1 ; \frac{1-2 x}{1-x}\right) d x \\
& =\frac{K}{c^{2}} \int_{0}^{1} \frac{z^{a-1}(1-z)^{a-1}}{(2-z)^{a+b+1}}{ }_{2} F_{1}(c, 1-b ; c+1 ; z) d z,
\end{aligned}
$$

where the transformation $z=(1-2 x) /(1-x)$ has been applied. Application of Lemma A.3 shows that the integral in (2.5) can be calculated to give

$$
R=\frac{K \Gamma(a+b) \Gamma(c+1)}{a c^{2} \Gamma(a+b+c)}{ }_{3} F_{2}(a, a+b+1, a+b ; a+1, a+b+c ;-1) .
$$

Elementary expressions for $R$ can be obtained if one assumes that $b$ or $c$ is an integer. If $b$ is an integer, then, by using the property

$$
I_{z}(\alpha, b)=\sum_{i=1}^{b} \frac{\Gamma(\alpha+i-1)}{\Gamma(\alpha) \Gamma(i)} z^{\alpha}(1-z)^{i-1}
$$

it follows from (2.3) that

$$
\begin{aligned}
R & =K B(c, b) \int_{0}^{1 / 2} x^{a-1}(1-x)^{b+c-1}\left\{\sum_{i=1}^{b} \frac{\Gamma(c+i-1)}{\Gamma(c) \Gamma(i)}\left(\frac{1-2 x}{1-x}\right)^{c}\left(\frac{x}{1-x}\right)^{i-1}\right\} d x \\
& =K B(c, b) \sum_{i=1}^{b} \frac{\Gamma(c+i-1)}{\Gamma(c) \Gamma(i)} \int_{0}^{1 / 2} x^{a-1}(1-x)^{b-i}(1-2 x)^{c} d x \\
& =K B(c, b) 2^{1-a} \sum_{i=1}^{b} \frac{\Gamma(c+i-1)}{2^{i} \Gamma(c) \Gamma(i)} \int_{0}^{1} y^{i+a-2}(1-y)^{c}\left(1-\frac{y}{2}\right)^{b-i} d y \\
& =K B(c, b) 2^{1-a} \sum_{i=1}^{b} \frac{\Gamma(c+i-1)}{2^{i} \Gamma(c) \Gamma(i)} \int_{0}^{1} y^{i+a-2}(1-y)^{c} \sum_{k=0}^{b-i}(-1)^{k}\left(\begin{array}{c}
b-i \\
k
\end{array}\right)\left(\frac{y}{2}\right)^{k} d y \\
& =K B(c, b) 2^{1-a} \sum_{i=1}^{b} \sum_{k=0}^{b-i}\left(\begin{array}{c}
b-i \\
k
\end{array}\right) \frac{(-1)^{k} \Gamma(c+i-1)}{2^{i+k} \Gamma(c) \Gamma(i)} \int_{0}^{1} y^{i+k+a-2}(1-y)^{c} d y \\
& =K B(c, b) 2^{1-a} \sum_{i=1}^{b} \sum_{k=0}^{b-i}\left(\begin{array}{c}
b-i \\
k
\end{array}\right) \frac{(-1)^{k} \Gamma(i+c-1) \Gamma(i+k+a-1)}{2^{i+k} \Gamma(i+k+a+c-1) \Gamma(i)},
\end{aligned}
$$

where the transformation $y=2 x$ has been applied. Thus, for integer values of $b$, one can express $R$ as a finite sum of elementary functions. On the other hand, if $c$ is an integer, 
104 Reliability for some bivariate beta distributions

then, by using the property

$$
I_{z}(c, \beta)=1-\sum_{i=1}^{c} \frac{\Gamma(\beta+i-1)}{\Gamma(\beta) \Gamma(i)} x^{i-1}(1-x)^{\beta}
$$

it follows from (2.3) that one can write

$$
\begin{aligned}
R & =K B(c, b) \int_{0}^{1 / 2} x^{a-1}(1-x)^{b+c-1}\left\{1-\sum_{i=1}^{c} \frac{\Gamma(b+i-1)}{\Gamma(b) \Gamma(i)}\left(\frac{1-2 x}{1-x}\right)^{i-1}\left(\frac{x}{1-x}\right)^{b}\right\} d x \\
& =K B(c, b)\left\{I-\sum_{i=1}^{c} \frac{\Gamma(b+i-1)}{\Gamma(b) \Gamma(i)} J(i)\right\},
\end{aligned}
$$

where

$$
\begin{aligned}
I & =\int_{0}^{1 / 2} x^{a-1}(1-x)^{b+c-1} d x \\
J(i) & =\int_{0}^{1 / 2} x^{a+b-1}(1-x)^{c-i}(1-2 x)^{i-1} d x .
\end{aligned}
$$

By the definition of the incomplete beta function ratio,

$$
I=B(a, b+c) I_{1 / 2}(a, b+c)
$$

and after transforming $y=2 x-J(i)$ can be calculated as

$$
\begin{aligned}
J(i) & =2^{-(a+b)} \int_{0}^{1} y^{a+b-1}(1-y)^{i-1}\left(1-\frac{y}{2}\right)^{c-i} d y \\
& =2^{-(a+b)} \int_{0}^{1} y^{a+b-1}(1-y)^{i-1}\left\{\sum_{k=0}^{c-i}(-1)^{k}\left(\begin{array}{c}
c-i \\
k
\end{array}\right)\left(\frac{y}{2}\right)^{k}\right\} d y \\
& =2^{-(a+b)} \sum_{k=0}^{c-i}(-2)^{-k}\left(\begin{array}{c}
c-i \\
k
\end{array}\right) \int_{0}^{1} y^{a+b+k-1}(1-y)^{i-1} d y \\
& =2^{-(a+b)} \sum_{k=0}^{c-i}(-2)^{-k}\left(\begin{array}{c}
c-i \\
k
\end{array}\right) B(a+b+k, i) .
\end{aligned}
$$

Substituting (2.12) and (2.13) into (2.10), one obtains the elementary expression

$$
R=K B(c, b)\left\{B(a, b+c) I_{1 / 2}(a, b+c)-2^{-(a+b)} \sum_{i=1}^{c} \sum_{k=0}^{c-i}\left(\begin{array}{c}
c-i \\
k
\end{array}\right) \frac{(-1)^{k} \Gamma(b+i-1) \Gamma(a+b+k)}{2^{k} \Gamma(b) \Gamma(a+b+i+k)}\right\}
$$

for integer values of $c$. 


\section{Connor and Mosimann's generalized Dirichlet distribution}

Connor and Mosimann generalized Dirichlet distribution [1] has the joint pdf given by

$$
f(x, y)=K x^{a-1} y^{b-1}(1-x)^{c-b-d}(1-x-y)^{d-1}
$$

for $x \geq 0, y \geq 0, x+y \leq 1, a>0, b>0, d>0$ and $c>b+d$, where

$$
K=\frac{\Gamma(a+c) \Gamma(b+d)}{\Gamma(a) \Gamma(b) \Gamma(c) \Gamma(d)} .
$$

For this distribution, $R$ can be expressed as

$$
\begin{aligned}
R & =K \int_{0}^{1 / 2} x^{a-1}(1-x)^{c-b-d} \int_{x}^{1-x} y^{b-1}(1-x-y)^{d-1} d y d x \\
& =K \int_{0}^{1 / 2} x^{a-1}(1-x)^{c-1} \int_{x /(1-x)}^{1} w^{b-1}(1-w)^{d-1} d w d x \\
& =K B(d, b) \int_{0}^{1 / 2} x^{a-1}(1-x)^{c-1} I_{(1-2 x) /(1-x)}(d, b) d x,
\end{aligned}
$$

where the transformation $w=y /(1-x)$ has been applied. Using the relationship (2.4), (3.3) can be rewritten as

$$
\begin{aligned}
R & =K \int_{0}^{1 / 2} x^{a-1}(1-x)^{c-d-1}(1-2 x)^{d}{ }_{2} F_{1}\left(d, 1-b ; 1+d ; \frac{1-2 x}{1-x}\right) d x \\
& =2^{-(a+c)} K \int_{0}^{1} z^{d}(1-z)^{a-1}(1-z / 2)^{-(c+a)}{ }_{2} F_{1}(d, 1-b ; 1+d ; z) d z
\end{aligned}
$$

which follows by transforming $z=(1-2 x) /(1-x)$. Now, an application of Lemma A.3 shows that (3.4) can be reduced to

$$
R=\frac{K \Gamma(d+1) \Gamma(a+b)}{a \Gamma(a+b+d)}{ }_{3} F_{2}(a, a+c, a+b ; a+1, a+b+d ;-1) .
$$

As in Section 2, simpler expressions for $R$ can be obtained for certain special cases. In particular, if $b$ is an integer, then, by using (2.7), one can show that

$$
R=\frac{K \Gamma(b)}{2^{a} \Gamma(b+d)} \sum_{i=1}^{b} \frac{\Gamma(d+i-1) B(a+i, d+1)}{2^{i} \Gamma(i)}{ }_{2} F_{1}\left(d-c+i, a+i ; a+d+i+1 ; \frac{1}{2}\right) .
$$

Also, if $d$ is an integer, then, by using (2.9), one can show that

$$
\begin{aligned}
R=K B(d, b)\{ & B(a, c) I_{1 / 2}(a, c)-\frac{\Gamma(a+b)}{2^{a+b} \Gamma(b)} \sum_{i=1}^{d} \frac{i \Gamma(b+i-1)}{\Gamma(a+b+i+1)} \\
& \left.\times{ }_{2} F_{1}\left(b-c+i+1, a+b ; a+b+i+1 ; \frac{1}{2}\right)\right\} .
\end{aligned}
$$




\section{Inverted Dirichlet distribution}

The inverted Dirichlet distribution has the joint pdf specified by

$$
f(x, y)=K \frac{x^{a-1} y^{b-1}}{(1+x+y)^{a+b+c}}
$$

for $x \geq 0, y \geq 0, a>0, b>0$, and $c>0$, where

$$
K=\frac{\Gamma(a+b+c)}{\Gamma(a) \Gamma(b) \Gamma(c)} .
$$

For this distribution, $R$ can be expressed as

$$
\begin{aligned}
R & =K \int_{0}^{\infty} x^{a-1} \int_{x}^{\infty} \frac{y^{b-1}}{(1+x+y)^{a+b+c}} d y d x \\
& =K \int_{0}^{\infty} \frac{x^{a-1}}{(1+x)^{a+b+c}} \int_{x}^{\infty} \frac{y^{b-1}}{(1+y /(1+x))^{a+b+c}} d y d x \\
& =\frac{K}{a+c} \int_{0}^{\infty} x^{-(c+1)}{ }_{2} F_{1}\left(a+b+c, a+c ; a+c+1 ;-\frac{1+x}{x}\right) d x
\end{aligned}
$$

where the last step follows by an application of Lemma A.4. On transforming $y=(1+$ $x) / x,(4.3)$ can be rewritten as

$$
R=\frac{K}{a+c} \int_{1}^{\infty}(y-1)^{c-1}{ }_{2} F_{1}(a+b+c, a+c ; a+c+1 ;-y) d y
$$

Now, the application of Lemma A.1 shows that (4.4) can be reduced to

$$
\begin{aligned}
R=\frac{K}{a+c}\{ & B(c,-c)_{3} F_{2}(a+b+c, a+c, 1 ; a+c+1, c+1 ;-1) \\
& \left.+\frac{\Gamma(c) \Gamma(a+b)}{a(a+c) \Gamma(a+b+c)}{ }_{3} F_{2}(1-c, a+b, a ; 1-c, a+1 ;-1)\right\} .
\end{aligned}
$$

An elementary expression for $R$ can be obtained if $b$ is an integer. In this case, using the property

$$
{ }_{2} F_{1}(\gamma-\alpha, \gamma-\beta ; \gamma ; z)=(1-z)^{\alpha+\beta-\gamma}{ }_{2} F_{1}(\alpha, \beta ; \gamma ; z)
$$

one can rewrite (4.4) as

$$
R=\frac{K}{a+c} \int_{1}^{\infty}(y-1)^{c-1}(y+1)^{1-a-b-c}{ }_{2} F_{1}(1-b, 1 ; a+c+1 ;-y) d y,
$$


which can be calculated, using the definition of the Gauss hypergeometric function, as

$$
\begin{aligned}
R & =\frac{K}{a+c} \int_{1}^{\infty}(y-1)^{c-1}(y+1)^{1-a-b-c} \sum_{k=0}^{b-1} \frac{(1-b)_{k}}{(a+c+1)_{k}}(-y)^{k} d y \\
& =\frac{K}{a+c} \sum_{k=0}^{b-1} \frac{(-1)^{k}(1-b)_{k}}{(a+c+1)_{k}} \int_{1}^{\infty} y^{k}(y-1)^{c-1}(y+1)^{1-a-b-c} d y \\
& =\frac{K}{a+c} \sum_{k=0}^{b-1} \frac{(-1)^{k}(1-b)_{k}}{(a+c+1)_{k}} \int_{0}^{\infty}(1+z)^{k} z^{c-1}(z+2)^{1-a-b-c} d z \\
& =\frac{K}{a+c} \sum_{k=0}^{b-1} \frac{(-1)^{k}(1-b)_{k}}{(a+c+1)_{k}} \int_{0}^{\infty}\left\{\sum_{l=0}^{k}\left(\begin{array}{l}
k \\
l
\end{array}\right) z^{l}\right\} z^{c-1}(z+2)^{1-a-b-c} d z \\
& =\frac{K}{a+c} \sum_{k=0}^{b-1} \sum_{l=0}^{k}\left(\begin{array}{l}
k \\
l
\end{array}\right) \frac{(-1)^{k}(1-b)_{k}}{(a+c+1)_{k}} \int_{0}^{\infty} z^{l+c-1}(2+z)^{1-a-b-c} d z \\
& =\frac{K}{2^{a+b-1}(a+c)} \sum_{k=0}^{b-1} \sum_{l=0}^{k}\left(\begin{array}{l}
k \\
l
\end{array}\right) \frac{(-1)^{k}(1-b)_{k} 2^{l}}{(a+c+1)_{k}} \int_{0}^{1} w^{c+l-1}(1-w)^{a+b-l-2} d w \\
& =\frac{K}{2^{a+b-1}(a+c)} \sum_{k=0}^{b-1} \sum_{l=0}^{k}\left(\begin{array}{l}
k \\
l
\end{array}\right) \frac{(-1)^{k}(1-b)_{k} 2^{l}}{(a+c+1)_{k}} B(c+l, a+b-l-1),
\end{aligned}
$$

where the transformations $z=y-1$ and $w=1 /(2+z)$ have been applied. A simpler expression than (4.5) can be obtained also if $a+c$ is an integer. In this case, by using the property that for $m>n$,

$$
\begin{aligned}
{ }_{2} F_{1}(n, \beta ; m ; z)= & \frac{z^{n-m}(m-1) ! \Gamma(\beta-m+n)}{(1-z)^{n-m+\beta}(n-1) ! \Gamma(\beta)} \sum_{k=0}^{n-1} \frac{(1-n)_{k}(m-n)_{k}}{(m-\beta-n+1)_{k} k !}\left(\frac{z-1}{z}\right)^{k} \\
& +\frac{z^{-n}(m-1) ! \Gamma(m-n-\beta)}{(m-n-1) ! \Gamma(m-\beta)} \sum_{k=0}^{m-n-1} \frac{(n-m+1)_{k}(n)_{k}}{(\beta-m+n+1)_{k} k !}\left(\frac{z-1}{z}\right)^{k},
\end{aligned}
$$

one can rewrite (4.7) as

$$
R=\frac{K(a+c-1) ! \Gamma(1-a-b-c)}{(-1)^{a+c} \Gamma(1-b)} I-\frac{K}{a+b+c} \sum_{k=0}^{a+c-1} \frac{(1-a-c)_{k}}{(2-a-b-c)_{k}} J(k)
$$

where

$$
\begin{gathered}
I=\int_{1}^{\infty} y^{-(a+c)}(y-1)^{c-1} d y, \\
J(k)=\int_{1}^{\infty} \frac{(y-1)^{c-1}}{y^{k+1}(y+1)^{a+b+c-k-1}} d y .
\end{gathered}
$$

On transforming $w=1 / y, I$ can be calculated as

$$
I=\int_{0}^{1} w^{c-1}(1-w)^{a-1} d w=B(c, a)
$$


108 Reliability for some bivariate beta distributions

and, on transforming $z=y-1, J(k)$ can be calculated as

$$
\begin{aligned}
J(k) & =\int_{1}^{\infty} \frac{z^{c-1}}{(1+z)^{k+1}(2+z)^{a+b+c-k-1}} d z \\
& =B(a+b, c)_{2} F_{1}(k+1-a-b-c, a+b ; a+b+c ;-1),
\end{aligned}
$$

where the last step follows by application of Lemma A.5. Substituting (4.12) and (4.13) into (4.10), one obtains the simpler expression

$$
\begin{aligned}
R= & \frac{K \Gamma(a) \Gamma(c) \Gamma(1-a-b-c)}{(-1)^{a+c} \Gamma(1-b)}-\frac{K B(a+b, c)}{a+b+c} \sum_{k=0}^{a+c-1} \frac{(1-a-c)_{k}}{(2-a-b-c)_{k}} \\
& \times{ }_{2} F_{1}(k+1-a-b-c, a+b ; a+b+c ;-1)
\end{aligned}
$$

for integer values of $a+c$.

\section{Lee's generalized inverted Dirichlet distribution}

Lee generalized inverted Dirichlet distribution [3] has the joint pdf given by

$$
f(x, y)=K \frac{(b k x)^{a-1}(b k y)^{a-1}}{(1+b k x)^{a+c}(1+b k y)^{a+c}} 2 F_{1}\left(a+c, a+c ; c ; \frac{1-k}{(1+b k x)(1+b k y)}\right)
$$

for $x \geq 0, y \geq 0, a>0, b>0, c>0$, and $k>0$, where

$$
K=\frac{b^{2} k^{c+2} \Gamma^{2}(a+c)}{\Gamma^{2}(a) \Gamma^{2}(c)}
$$

Using the definition of the Gauss hypergeometric function, the corresponding form of $R$ can be expressed as

$$
R=K(b k)^{2 a-2} \sum_{m=0}^{\infty} \frac{(a+c)_{m}(a+c)_{m}(1-k)^{m}}{(c)_{m} m !} I(m)
$$

where

$$
I(m)=\int_{0}^{\infty} \frac{x^{a-1}}{(1+b k x)^{a+c+m}} \int_{x}^{\infty} \frac{y^{a-1}}{(1+b k y)^{a+c+m}} d y d x
$$

An application of Lemma A.4 reduces this integral to

$$
\begin{aligned}
I(m) & =\frac{(b k)^{-(a+c+m)}}{c+m} \int_{0}^{\infty} \frac{x^{a-c-m-1}}{(1+b k x)^{a+c+m}}{ }_{2} F_{1}\left(a+c+m, c+m ; c+m+1 ;-\frac{1}{b k x}\right) d x \\
& =\frac{(b k)^{-2 a}}{c+m} \int_{0}^{\infty} \frac{y^{2 a+2 m-1}}{(1+y)^{a+c+m}}{ }_{2} F_{1}(a+c+m, c+m ; c+m+1 ;-y) d y,
\end{aligned}
$$


where the last step follows after transforming $y=1 /(b k x)$. The integral in (5.5) can be calculated by a direct application of Lemma A.2; so, it follows from (5.3) that

$$
\begin{aligned}
R=\frac{K}{(b k)^{2}} \sum_{m=0}^{\infty} & \frac{(a+c)_{m}(a+c)_{m}(1-k)^{m}}{(c+m)(c)_{m} m !} \\
& \times\left\{\begin{array}{l}
B(2 c+2 m, a-c-m) \\
\quad
\end{array}\right. \\
& \quad+\frac{(c+m) \Gamma(2 a) \Gamma(c+m-a)}{a \Gamma(a+c+m)}{ }_{3} F_{2}(a+c+m, c+m, 2 c+2 m ; c+m+1, c+m-a+1 ; 1) \\
&
\end{aligned}
$$

As in Section 4, simpler expressions for $R$ can be obtained in certain special cases. In particular, if $a$ is an integer, then, using (4.6), one can show that

$$
R=\frac{K}{(b k)^{2}} \sum_{m=0}^{\infty} \sum_{n=0}^{a-1} \frac{(-1)^{n}(a+c)_{m}(a+c)_{m}(1-k)^{m}(1-a)_{n}}{(c+m)(c)_{m}(c+m+1)_{n} m !} B(2 c+2 m+n, 2 a-n-1) .
$$

Also, if $c$ is an integer, then, using (4.9), one can show that

$$
\begin{aligned}
R=\frac{K}{(b k)^{2}} \sum_{m=0}^{\infty} & \frac{(a+c)_{m}(a+c)_{m}(1-k)^{m}}{(c+m)(c)_{m} m !} \\
& \times\left\{\frac{\Gamma(a) \Gamma^{2}(c+m) \Gamma(1-a-c-m)}{(-1)^{c+m} \Gamma(a+c+m) \Gamma(1-a)}\right. \\
& \left.\quad-\frac{1}{a+c+m-1} \sum_{n=0}^{c+m-1} \frac{(1-c-m)_{n}}{(2-a-c-m)_{n}} B(2 c+2 m-n-1,2 a)\right\} .
\end{aligned}
$$

\section{Beta Stacy distribution}

The beta Stacy distribution (Mihram and Hultquist [4]) has the joint pdf specified by

$$
f(x, y)=\frac{|c|}{a^{b c} \Gamma(b) B(p, q)} x^{p-1} y^{b c-p-q}(y-x)^{q-1} \exp \left\{-\left(\frac{y}{a}\right)^{c}\right\}
$$

for $y>x \geq 0, a>0, b>0,-\infty<c<\infty, p>0$, and $q>0$. By definition, it is clear that $R=1$. 
110 Reliability for some bivariate beta distributions

\section{Appendix}

Some technical lemmas required for the calculations above are noted below.

Lemma A.1 (Prudnikov et al. [13, equation (2.21.1.13)]). For $\alpha>0$ and $\beta>0$,

$$
\begin{aligned}
& \int_{y}^{\infty} x^{\alpha-1}(x-y)^{\beta-1}{ }_{2} F_{1}(a, b ; c ;-w x) d x \\
&= B(\beta, 1-\alpha-\beta) y^{\alpha+\beta-1}{ }_{3} F_{2}(a, b, \alpha ; c, \alpha+\beta ;-w y) \\
&+K w^{1-\alpha-\beta}{ }_{3} F_{2}(1-\beta, a-\alpha-\beta+1, b-\alpha-\beta+1 ; 2-\alpha-\beta, c-\alpha-\beta+1 ;-w y),
\end{aligned}
$$

where

$$
K=\frac{\Gamma(c) \Gamma(a-\alpha-\beta+1) \Gamma(b-\alpha-\beta+1) \Gamma(\alpha+\beta-1)}{\Gamma(a) \Gamma(b) \Gamma(c-\alpha-\beta+1)} .
$$

Lemma A.2 (Prudnikov et al. [13, equation (2.21.1.15)]). For $\alpha>0$,

$$
\begin{gathered}
\int_{0}^{\infty} x^{\alpha-1}(x+z)^{-\rho}{ }_{2} F_{1}(a, b ; c ;-w x) d x \\
=z^{\alpha-\rho} B(\alpha, \rho-\alpha)_{3} F_{2}(a, b, \alpha ; c, \alpha-\rho+1 ; w z) \\
+K w^{\rho-\alpha}{ }_{3} F_{2}(a-\alpha+\rho, b-\alpha+\rho, \rho ; c-\alpha+\rho, \rho-\alpha+1 ; w z), \\
K=\frac{\Gamma(c) \Gamma(a-\alpha+\rho) \Gamma(b-\alpha+\rho)}{\Gamma(a) \Gamma(b) \Gamma(c-\alpha+\rho)} .
\end{gathered}
$$

Lemma A.3 (Prudnikov et al. [13, equation (2.21.1.22)]). For $c>0$ and $\beta>0$,

$$
\begin{aligned}
& \int_{0}^{y} \frac{x^{c-1}(y-x)^{\beta-1}}{(1-z x)^{\rho}}{ }_{2} F_{1}\left(a, b ; c ; \frac{x}{y}\right) d x \\
& \quad=\frac{K y^{c+\beta-1}}{(1-y z)^{\rho}}{ }_{3} F_{2}\left(\beta, \rho, c-a-b+\beta ; c-a+\beta, c-b+\beta ; \frac{y z}{y z-1}\right),
\end{aligned}
$$

where

$$
K=\frac{\Gamma(c) \Gamma(\beta) \Gamma(c-a-b+\beta)}{\Gamma(c-a+\beta) \Gamma(c-b+\beta)} .
$$

Lemma A.4 (Gradshteyn and Ryzhik [2, equation (3.194.2)]). For $v>\mu>0$,

$$
\int_{u}^{\infty} \frac{x^{\mu-1}}{(1+\beta x)^{\nu}} d x=\frac{u^{\mu-\nu}}{\beta^{\nu}(\nu-\mu)}{ }_{2} F_{1}\left(\nu, \nu-\mu ; \nu-\mu+1 ;-\frac{1}{\beta u}\right) .
$$

Lemma A.5 (Gradshteyn and Ryzhik [2, equation (3.197.9)]). For $\mu>\lambda>0$,

$$
\int_{0}^{\infty} x^{\lambda-1}(1+x)^{-\mu+\nu}(x+\beta)^{-v} d x=B(\mu-\lambda, \lambda)_{2} F_{1}(\nu, \mu-\lambda ; \mu ; 1-\beta) .
$$




\section{Acknowledgments}

The author is grateful to Professor Samuel Kotz (George Washington University, USA) and to Professor Marianna Pensky (University of Central Florida, USA) for introducing him to this area of research.

\section{References}

[1] R. J. Connor and J. E. Mosimann, Concepts of independence for proportions with a generalization of the Dirichlet distribution, J. Amer. Statist. Assoc. 64 (1969), 194-206.

[2] I. S. Gradshteyn and I. M. Ryzhik, Table of Integrals, Series, and Products, Academic Press, California, 2000.

[3] P. A. Lee, The correlated bivariate inverted beta distribution, Biometrical J. 23 (1981), no. 7, 693-703.

[4] G. A. Mihram and R. A. Hultquist, A bivariate warning-time/failure-time distribution, J. Amer. Statist. Assoc. 62 (1967), 589-599.

[5] S. Nadarajah, Reliability for beta models, Serdica Math. J. 28 (2002), no. 3, 267-282.

[6] Reliability for extreme value distributions, Math. Comput. Modelling 37 (2003), no. 910, 915-922.

[7] _ Reliability for lifetime distributions, Math. Comput. Modelling 37 (2003), no. 7-8, 683688.

[8] _ Reliability for Laplace distributions, Math. Probl. Eng. 10 (2004), 169-183.

[9] _ Reliability for logistic distributions, Engineering Simulation 26 (2004), 81-98.

[10] S. Nadarajah and S. Kotz, Reliability for Pareto models, Metron 61 (2003), no. 2, 191-204.

[11] A. P. Prudnikov, Y. A. Brychkov, and O. I. Marichev, Integrals and Series. Vol. 1, Gordon \& Breach Science Publishers, New York, 1986.

[12] _ Integrals and Series. Vol. 2, Gordon \& Breach Science Publishers, New York, 1986.

[13] _ Integrals and Series. Vol. 3, Gordon \& Breach Science Publishers, New York, 1990.

Saralees Nadarajah: Department of Statistics, University of Nebraska, Lincoln, NE 68583, USA

E-mail address: snadaraj@math.iupui.edu 


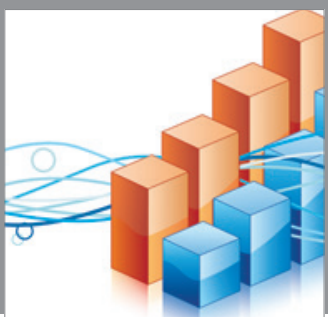

Advances in

Operations Research

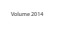

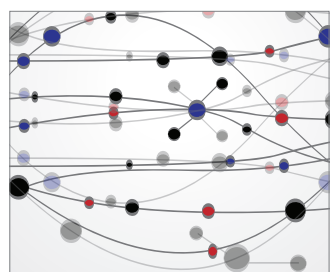

\section{The Scientific} World Journal
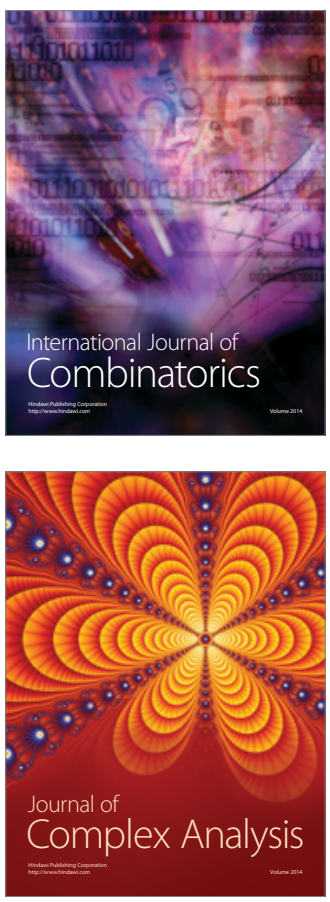

International Journal of

Mathematics and

Mathematical

Sciences
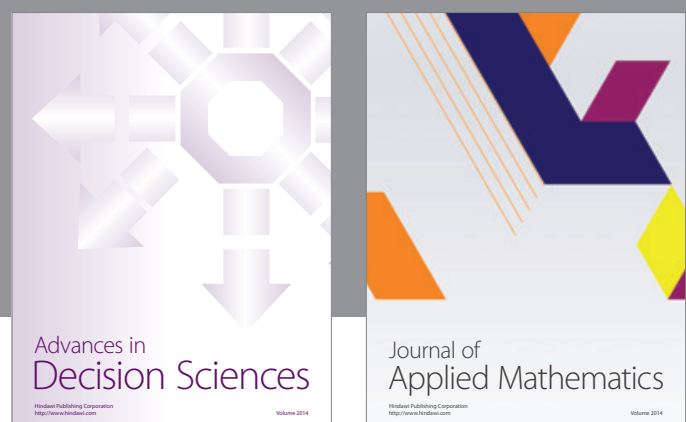

Journal of

Applied Mathematics
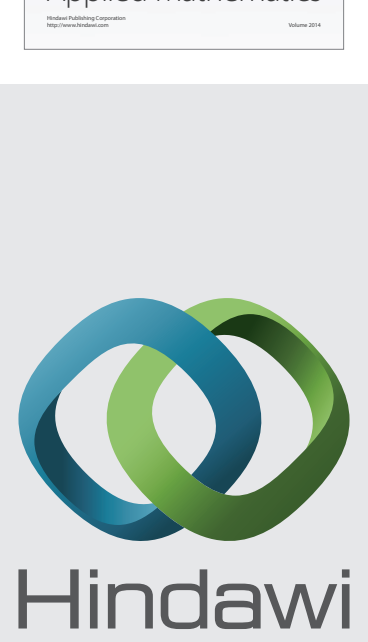

Submit your manuscripts at http://www.hindawi.com
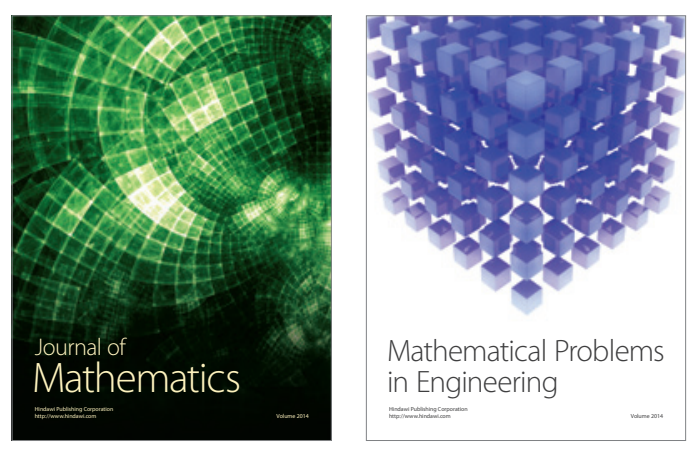

Mathematical Problems in Engineering
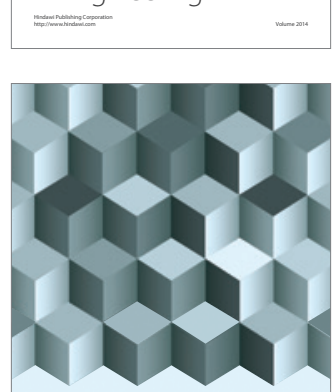

Journal of

Function Spaces
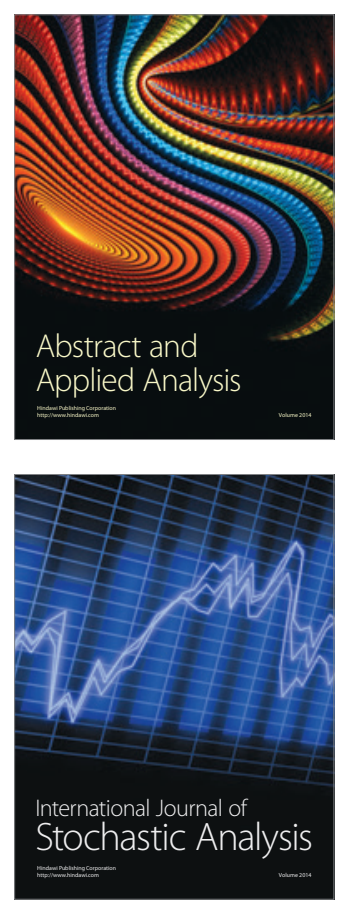

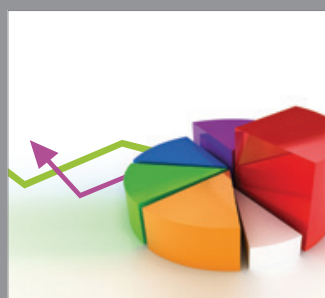

ournal of

Probability and Statistics

Promensencen
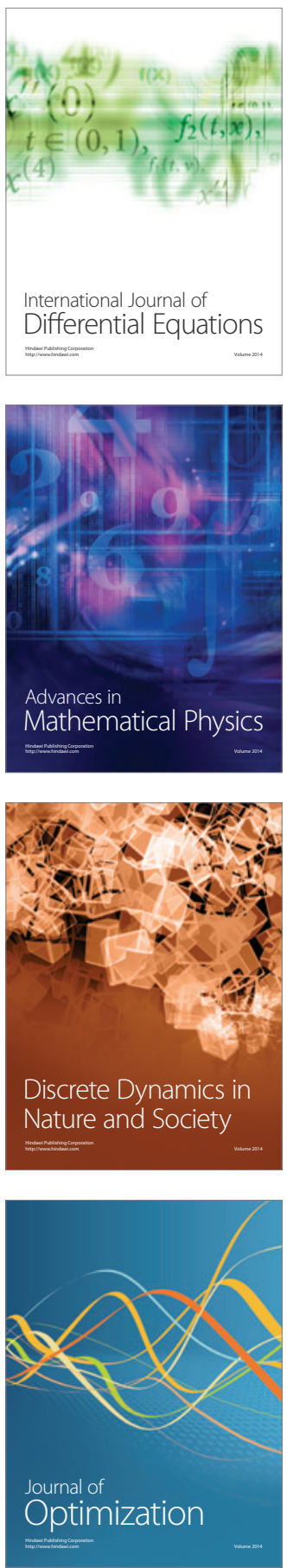